ISSN 2179-6750

\title{
Práticas integrativas e complementares e racionalidades médicas no SUS e na atenção primária à saúde: possibilidades estratégicas de expansão
}

\author{
Complementary and alernative medicina and medical rationalities in SUS and in primary health \\ care: strategic possibilities for expansion \\ Prácticas complementarias e integradoras y racionalidades médicas en el SUS y en la atención \\ primaria de salud: posibilidades de expansión
}

\section{Charles Dalcanale Tesser ${ }^{1}$}

\section{Resumo}

Discutimos a expansão das práticas integrativas e complementares (PIC) no Sistema Único de Saúde (SUS), objetivando contribuir para o desenvolvimento de diretrizes gerais orientadoras dos profissionais e gestores. Após a edição da Política Nacional de PIC (PNPIC), cuja única diretriz concreta de implantação é a ênfase na atenção primária à saúde (APS), a inserção dessas práticas tem sido errática. Os municípios (e às vezes serviços) têm sua experiência própria, sem apoio técnico ou financeiro e sem orientação institucional. Discutimos os principais modos dessa inserção na APS e em outros serviços do SUS. Nossa argumentação discute possibilidades estratégicas de expansão das PIC derivadas dessas experiências. Argumentamos pelo reconhecimento da APS como lócus privilegiado de inserção das PIC. Concluímos que a inserção das PIC no SUS deve privilegiar enfaticamente a APS através de seus profissionais generalistas. Para isso há que desenvolver estratégias de educação permanente e de formação que as tirem da situação de marginalidade. Por outro lado, há que incentivar também sua presença em serviços especializados, sobretudo as racionalidades médicas vitalistas, cuja profundidade e complexidade sugerem o desenvolvimento de centros de expertise nas instituições formadoras e no SUS, atuando com matriciamento de equipes de APS, para que contribuam com cuidado especializado aos usuários e educação permanente dos profissionais da APS.

Descritores: Terapias Complementares; Atenção Primária à Saúde; Sistema Único de Saúde.

\section{Abstract}

We discuss the expansion of integrative and complementary practices (PIC) in the Unified Health System (SUS), aiming to contribute to the development of general guidelines for professionals and managers. Following the publication of the National PIC Policy (PNPIC), whose only concrete implementation guideline is the emphasis on primary health care (PHC), the insertion of these practices has been erratic. Municipalities (and sometimes services) have their own experience, without technical or financial support and without institutional guidance. We discuss the main modes of this insertion in PHC and other SUS services. Our argument discusses strategic possibilities for expansion of PIC derived from these experiences. We argue for the recognition of APS as a privileged locus of insertion of PIC. We conclude that the insertion of PIC into SUS should emphatically favor PHC through its general practitioners. For this purpose, strategies for permanent education and training must be developed to remove them from the situation of

\footnotetext{
${ }^{1}$ Médico, doutor em Saúde Coletiva pela Universidade Estadual de Campinas (UNICAMP). Professor Adjunto do Departamento de Saúde Pública - Centro de Ciências da Saúde - Universidade Federal de Santa Catarina (UFSC). Campus Universitário, Trindade, Florianópolis, SC, Brasil. CEP: 88040-900. E-mail: charles.tesser@ufsc.br
} 
ISSN 2179-6750

marginality. On the other hand, their presence in specialized services, especially the vitalist medical rationalities, whose depth and complexity suggest the development of centers of expertise in the training institutions and in the SUS, should be encouraged, working with the training of PHC teams, to contribute to specialized care to users and ongoing education of PHC professionals.

Key-words: Complementary Therapies; Primary Health Care; Unified Health System.

\section{Resumen}

Discutimos la expansión de las prácticas complementarias y integradoras (PIC) en el Sistema Único de Salud (SUS), con el objetivo de contribuir al desarrollo de directrices generales para los profesionales y gestores. Después de editar la política nacional de PIC (PNPIC), cuya única pauta concreta es el énfasis en la atención primaria de salud (APS), la inclusión de estas prácticas ha sido errático. Municipios (ya veces los servicios) tienen su propia experiencia, sin apoyo técnico o financiero, ni dirección institucional. Se discuten los principales modos de esta inclusión en la APS y otros servicios del SUS. Examinamos las oportunidades estratégicas para la expansión de la PIC derivadas de estas experiencias. Argumentamos por el reconocimiento de la APS como un lugar privilegiado de la inserción de las PIC. Llegamos a la conclusión de que la inclusión de PIC en el SUS debe favorecer fuertemente la APS a través de sus médicos de cabecera. Para ello, es necesario desarrollar estrategias de aprendizaje permanente y la formación para que las PIC se cambiem de la situación de marginación. Por otro lado, también debe fomentar su presencia en servicios especializados, especialmente las racionalidades médicas, cuya profundidad y complejidad sugieren el desarrollo de centros especializados en las instituciones educativas y el SUS, para trabajar matriciando equipos de APS y llevar a cabo la atención especializada y la formación continua de PIC.

Palabras-claves: Terapias Complementarias; Atención Primaria de Salud; Sistema Único de Salud.

\section{Introdução}

No Brasil, o Ministério da Saúde designou como Práticas Integrativas e Complementares (PIC) o que na literatura biomédica e de saúde pública tem se chamado de medicinas alternativas e complementares. A organização mundial da saúde (OMS) tem apoiado a institucionalização dessas práticas nos sistemas públicos de saúde ${ }^{1}$. Em geral, elas são pragmaticamente reconhecidas e agrupadas por não pertencerem ao escopo da medicina convencional ${ }^{1}$, embora constituam um conjunto muito heterogêneo. Usaremos neste artigo a expressão PIC devido a sua comum utilização pelos gestores e profissionais do SUS.

Madel Luz ${ }^{2}$ distinguiu e especificou, dentro da heterogeneidade das PIC, os sistemas médicos complexos, visando seu estudo e comparação. Para isso construiu a categoria racionalidade médica (RM), à moda de um tipo ideal weberiano, definida como um conjunto integrado, articulado e coerente de seis dimensões: uma cosmologia, uma doutrina médica, uma dinâmica vital, uma morfologia humana, um sistema de diagnose e um de terapêutica. Através dela, desenvolveu estudos de sistemas médicos que apresentam essas seis dimensões suficientemente elaboradas e desenvolvidas (constituindo por isso racionalidades médicas), incluindo a homeopatia, a medicina 
ISSN 2179-6750

chinesa tradicional, a biomedicina, a medicina antroposófica e a medicina ayurvédica, sem usar a biomedicina e seus saberes como referência ou parâmetro de verdade. Constatou que todas, com exceção da biomedicina, compartilham características vitalistas, associadas a cosmologias integradoras do homem com a natureza ou o universo e a concepções energéticas do processo saúde-doença-atenção, em que a promoção da saúde e o cuidado nos adoecimentos envolvem o reequilíbrio e a estimulação de potenciais endógenos de autocura, em que a participação das pessoas, conforme as técnicas de cada racionalidade, é não só possível como estimulada. Neste texto, nos referiremos a elas como RM vitalistas, um subgrupo das PIC que hora é identificado como uma prática (um tratamento homeopático, por exemplo), hora é tratado como um sistema amplo que abrange várias práticas terapêuticas ou de promoção de saúde, em geral associadas entre si (como a medicina chinesa tradicional, que inclui massagem, acupuntura, medicamentos com substâncias vegetais, animais e minerais, práticas energéticas/corporais e de meditação etc.).

Devido ao seu caráter de maior complexidade, mais ampla e sofisticada abordagem, envolvendo diagnose e terapêutica próprias inseridas em saberes e valores alicerçados em cosmovisões distintas, algumas com muitos séculos, essas RM podem ser considerados como outros paradigmas, em sentido semelhante ao proposto por Kuhn ${ }^{3,4}$; ou, mais propriamente, outros estilos de pensamento, conforme Fleck ${ }^{5}$. Isso significa que as interpretações dos adoecimentos e seu manejo (tratamentos) nessas RM são radicalmente distintas das interpretações biomédicas, e seu aprendizado e exercício envolvem longa iniciação e relativa especialização. No dizer de Fleck ${ }^{5}$, há círculos esotéricos (especializados) de praticantes e produtores/reprodutores de saber, acessíveis através de longo processo de iniciação, o que implica em imersão nas perspectivas, nos valores, nas técnicas, nas habilidades perceptivas e cognitivas e nas cosmovisões particulares de cada RM.

Todavia, vale lembrar que se os profissionais de saúde, em geral (inclusive da APS), devem ser considerados membros iniciados nos estratos especializados e esotéricos de uma RM (no caso, biomédica), eles, por outro lado, estão na periferia desses círculos esotéricos e são influenciados pelos círculos, valores e saberes exotéricos, da sociedade e de seus usuários (considerados então leigos) ${ }^{6}$. Isso significa que na prática, os profissionais de saúde situam-se numa situação intermediária entre os super-especialistas de sua RM e os leigos. No caso da APS, é natural e amplamente consensual na literatura mundial que os profissionais ali atuantes sejam generalistas ${ }^{7}$ (mesmo que só do ponto de vista da biomedicina). Ou seja, que tenham uma formação e competência geral de modo a poder oferecer e adaptar o que interessa e é importante dos saberes especializados e superespecializados da RM biomédica para o cuidado clínico-sanitário comunitário, individual e coletivo. Nesse sentido, há uma contextualização e certo pragmatismo na 
ISSN 2179-6750

seleção dos saberes especializados, adaptando-se os mesmos, o que implica grande profundidade em alguns saberes/técnicas e mínima ou nenhuma profundidade em outros, úteis em outros contextos de cuidado que não a APS. De certo modo, um raciocínio análogo a esse pode ser aplicado às RM vitalistas no que tange à APS, e mesmo às PIC em geral. Essas considerações iniciais serão úteis mais adiante na discussão da inserção e expansão das PIC no SUS, em que as RM merecem, por essas características acima mencionadas, algumas considerações específicas.

Por outro lado, várias PIC podem ser e comumente são usadas em contexto sincréticos de cuidado, como é o caso do cuidado doméstico ou autoatenção ${ }^{8}$. Esse também é aproximadamente o caso dos serviços de atenção primária à saúde (APS), que de regra são dominados largamente pela RM da biomedicina, mas cuja inserção comunitária, quando concretiza os atributos da APS propostos por Starfield ${ }^{7}$ (acesso facilitado universal, integralidade, longitudinalidade, coordenação do cuidado, abordagem familiar e comunitária com competência cultural), demanda uma aproximação significativa com outros valores e práticas de cuidado presentes nas comunidades em que estão situados. Nesses ambientes, comumente há a tendência pragmática de uso de recursos e técnicas terapêuticas adaptadas às circunstâncias, situações, valores e preferências dos usuários, suas concepções de mundo e suas possibilidades sociais, econômicas e existenciais. Nesses casos (autoatenção e APS) são um tanto atenuadas as divergências doutrinárias, paradigmáticas e interpretativas em prol de uma pragmática de benefícios aos usuários, de práticas aceitáveis social e institucionalmente e de resultados percebidos pelos envolvidos. Em outras palavras, há no ambiente da APS uma maior interação dos profissionais com as racionalidades leigas ${ }^{9,10}$ que são desenvolvidas pelas pessoas e as orientam nos seus itinerários e práticas terapêuticas, bem como com saberes e práticas não biomédicos. Na APS várias PIC podem ser utilizadas sem o aprofundamento e a competência perceptiva e interpretativa supostos na abordagem especializada profissional orientada por uma RM, e inclusive as traduções e aproximações parciais não raro bastam para satisfazer as visões, crenças e necessidades de respostas de usuários e profissionais.

Um exemplo didático é o uso da auriculoterapia na APS. Uma prática que consiste no estímulo auricular comumente através de sementes esféricas coladas com um adesivo em pontos específicos da orelha. Tal prática, antiga tanto no ocidente quanto no oriente ${ }^{11}$, pode ser entendida, interpretada e realizada conforme a RM da medicina chinesa tradicional (com seus saberes envolvendo yin e yang, meridianos, cinco movimentos ou elementos ${ }^{12}$ ); conforme a reflexologia da orelha, desenvolvido por Nogier na década de $1940-50^{13}$, com a ideia de que todo o corpo está representado no (e conectado com) o pavilhão auricular; e conforme a biomedicina, ao menos parcialmente, que dispõem de modelos experimentais, hipóteses teóricas neurofisiológicas e 
ISSN 2179-6750

evidências sistematizadas (ensaios clínicos) sobre segurança e eficácia dessa prática ${ }^{14}$, comumente usada como recurso complementar de tratamento, ou único em casos menos graves. Esse é um exemplo de uma técnica simples, rápida e barata que é aceitável social e institucionalmente a partir de saberes relativamente simples (vinculados ao mapa de pontos auriculares em que está representado o corpo todo) e percepções largamente convergentes com as evidências sobre a eficácia e a segurança. Tal exemplo mostra que essa prática:

a) é amplamente aceitável no cuidado autônomo e institucional;

b) tem eficácia empírica, tanto nas tradições da RM chinesa tradicional, quanto nos ensaios clínicos realizados (RM biomédica), quanto percebida pelos usuários que dela usufruem;

c) persiste firme e forte na sua potência e nos seus limites terapêuticos, de forma relativamente imune à polêmica ou disputa teórica sobre quais as explicações "verdadeiras" sobre seu funcionamento;

d) exemplifica a possibilidade de uma apropriação de saber, capacitação técnica e consequente aumento de competência terapêutica que, ao mesmo tempo que inicia os praticantes em lógicas e saberes especializados que subjazem à auriculoterapia, não os subjulga nem os escraviza no sentido de ter que acreditar nas teorias chinesas, ou na reflexologia da orelha, ou virar especialista em neurofisiologia e medicina baseada em evidências, para poder aprender e usar esse recurso na APS;

e) contribui para ampliar a visão e a abordagem dos usuários pelos profissionais de saúde da APS, pluralizando as possibilidades de sua ação, independentemente dos diagnósticos biomédicos, os quais, em certa medida e pelo menos em vários casos e situações, pouco fazem diferença para a auriculoterapia;

f) chama a atenção para as múltiplas formas de se estimular e modular as funções e sensações dos organismos e das pessoas, num sentido de harmonia e bem-estar;

g) retrata e desnuda a possibilidade de ações de cuidado envolvendo estímulos aos mecanismos endógenos ("naturais") de regulação, regeneração e autocura, via de ação das RM vitalistas e de várias PIC, reconhecidamente um dos maiores méritos de várias delas ${ }^{15}$. Isso é particularmente importante devido a uma matriz cultural derivada do iluminismo, da biociência e da revolução científica e industrial, hoje sustentada pela mídia, que permeia o mundo moderno e disseminou entre nós ocidentais (pelo menos) um pano de fundo de valores e pressupostos que desvalorizam constantemente e profundamente as potências naturais autóctones de regulação, adaptação, regeneração e autocura do ser humano e da natureza; infundindo medo e pessimismo quanto ao fluxo natural dos acontecimentos, incluindo os processos saúde-doença (que precisariam 
ISSN 2179-6750

e poderiam ser controlados pela ação humana, entendido o humano não como parte de uma natureza maior e generosa, mas como senhor e imperador a dominar, controlar e manipular a natureza, interna e externa ${ }^{16}$ ). Tal fundo de valores e crenças hipertrofia desmesuradamente a suposta potência ilimitada da ciência e da tecnologia para resolver todos os problemas de saúde-doença, a depender cada vez mais de uma aparelhagem sofisticada e cara na qual são projetadas quase todas as potências de cura e às vezes mesmo de cuidado, inclusive gerando novas necessidades mediadas somente por esses aparelhos ${ }^{16}$. Obviamente, isso gera e reforça o processo de biomedicalização ${ }^{17}$ intensa que se desenrola nas sociedades industrializadas, gerando grande consumo de tecnologias duras (exames diagnósticos, fármacos, cirurgias), não só para o tratamento, mas também, recentemente, para a prevenção. Esse processo torna mais relevante e significativa a presença das PIC no SUS, supondo que elas podem contribuir sobremaneira para a pluralização das interpretações dos problemas de saúde e das possibilidades de seu manejo, ampliando as opções terapêuticas e promocionais para o cuidado dos usuários.

\section{Locais institucionais estratégicos para inserção das PIC}

Esses preâmbulos introdutórios preparam o terreno para a discussão principal sobre possibilidades estratégicas e construção de diretrizes para expansão das PIC no SUS. Primeiramente, vale a pena reiterar vários motivos pelos quais deve ser enfatizada a correção da única diretriz até agora vigente no SUS para as PIC: a prioridade para a atenção básica.

Para além de três quartos das PIC do SUS estarem na APS, deve ser enfatizado que é ali que elas devem estar. Ou, ao menos, é ali na APS que elas têm o maior potencial de contribuição ao SUS. São vários os motivos para isso e elencamos sinteticamente alguns deles: a) sua notória capacidade de estimular o potencial de autocura do sujeitos ${ }^{15}$, por hipótese, muito mais efetivo e potente se utilizada em situações iniciais ou menos graves dos adoecimentos, o que é justamente o caso do contexto comunitário em que a APS se insere; b) sua reconhecida aceitação pelos vários grupos sociais, derivada de vários fatores, dentre eles, a multiplicidade de suas raízes culturais, a convergência de seus valores e concepções de mundo e adoecimento ${ }^{18}$; c) o reconhecido bom relacionamento com os usuários, envolvendo uma melhor ressignificação e experiência dos adoecimentos e cuidados, com maior participação dos doentes, o que é sobretudo valioso e importante em situações de adoecimento crônico ${ }^{19}$; d) a progressiva importância e maior prevalência de problemas crônicos, devido a maior longevidade da população, que torna muito relevante a participação das pessoas e sua adaptação às transformações envolvidas nos adoecimentos crônicos e seu manejo - o que, por outro lado, gera no cuidado biomédico maior 
ISSN 2179-6750

iatrogenia, motivadora da busca por alternativas de tratamento, um dos grandes motivos de procura das PIC; e) o caráter menos iatrogênico em geral dessas práticas, comparativamente à biomedicina; f) (decorrente dos anteriores) o potencial de redução de danos e de facilitação de manejo e redução de excessos de intervenção, medicalização e iatrogenia, presente nas PIC em geral e nas RM vitalistas em particular ${ }^{20}$; g) sua potencial contribuição para uma ampliação da capacidade interpretativa e terapêutica com segurança e baixo risco para muitas situações de adoecimento inespecíficas e pouco enquadráveis na nosologia biomédica, que respondem por uma substancial proporção do volume de atendimentos dos serviços de APS ${ }^{21}$, e muito mais presentes na APS que em outros ambientes de cuidado institucional; h) uma maior aproximação dos terapeutas das vivências dos doentes e um maior compartilhamento simbólico com os usuários, que facilita o fluxo de orientações e cuidados, o acolhimento e a empatia exercida pelos profissionais, dado que uma das frustrações dos usuários com a biomedicina é o comum tecnicismo materialista da abordagem dos profissionais, com pouca escuta ${ }^{22}$, impessoalidade, pouca atenção aos aspectos subjetivos ${ }^{23} \mathrm{e}$ relativamente pobre manejo das singularidades dos doentes (relegados a arte da prática médica e dos outros profissionais), além de divergências sobre a natureza dos adoecimentos ${ }^{24}$.

Em síntese, múltiplos fatores de várias ordens convergem para se pensar que as PIC devem ser inseridas prioritariamente na APS, sendo ali o lócus ao mesmo tempo maximizador de sua potência e facilitador de seu ação.

Todavia, várias PIC e as RM vitalistas, sobretudo nas doenças crônicas, têm sido muito procuradas e mais estudadas em situações de maior gravidade e contextos de cuidados biomédicos especializados. Isso tanto pela procura dos doentes quanto pelas limitações terapêuticas biomédicas, que nesses casos pressionam por uma maior exploração de alternativas (vide o surgimento de áreas como a oncologia integrativa ${ }^{25}$, por exemplo) e demandam uma maior atenção às pessoas, à sua qualidade de vida e a seus valores (como na medicina paliativa), induzindo uma maior abertura para as PIC. Esses pacientes, mais graves em geral do que o grosso dos pacientes manejados apenas dentro da APS, são uma pequena parcela dos usuários do SUS. Por isso, a inserção das PIC nesses ambientes pode ser pensada como mais restrita e de pouco impacto, pois além de proporcionalmente poucos, os seus beneficiários teriam um menor benefício suposto, dado que os seus adoecimentos já são bem mais graves ou avançados, em situações nas quais as PIC entram como recursos complementares, esgotados os tratamentos biomédicos ou paralelos a estes, menos reconhecidas pelas suas potências terapêuticas e mais como recursos de melhoria de qualidade de vida (embora em vários casos as suas eficácias terapêuticas sejam progressivamente consideradas relevantes, conforme avançam as pesquisas). 
ISSN 2179-6750

Por isso seria defensável a construção de polos de pesquisa, prática especializada e reprodução de saber de PIC e especialmente das RM vitalistas. Tais polos poderiam atuar como referências de atendimento e ensino (graduação, pós-graduação e educação permanente) dessas RM, dada a escassez de sua institucionalização nas instituições de pesquisa e formação dos profissionais de saúde, sobretudo da APS.

Concluindo, podemos considerar que várias PIC e RM vitalistas devem mesmo ser prioritariamente inseridas na APS, mas também faz sentido e é defensável, pelos argumentos acima, que sejam inseridas em ambientes especializados. Trata-se, então, de desdobrar a seguir essas duas ideias para o contexto concreto do SUS e de ponderar, à luz da experiência acumulada no país, como articular da melhor maneira e em coerência com a construção difícil do SUS (entendido aqui como rede de servidos coordenada por uma APS resolutiva de acesso universal) as formas mais promissoras de inserção das PIC e RM vitalistas.

\section{Modos institucionais e administrativos de inserção}

Uma pesquisa ${ }^{26}$ mapeou quatro modos mais comuns de inserção das PIC no SUS em cinco grandes municípios, e nas linhas que seguem desenvolvemos algumas considerações sobre os mesmos. O primeiro modo de inserção das PIC é através dos próprios profissionais da APS, que as praticam. Supõem-se, nesse caso, que eles têm competência para tal, ou por formação própria prévia (em geral em paralelo com sua prática profissional no SUS) ou por formação em serviço, quando há iniciativas institucionais (geralmente municipais) de educação em serviço voltadas para as PIC.

O segundo modo ocorre quando há profissionais na APS que só praticam alguma PIC ou RM vitalista (mais comum: acupuntura, homeopatia), em paralelo aos seus colegas que realizam o cuidado biomédico.

O terceiro modo se dá quando há matriciamento em PIC, via núcleos de apoio à saúde da família (NASF) ou outras equipes de apoio. O quarto ocorre quando profissionais de alguma PIC atuam em serviços especializados biomédicos ou hospitalares, ou serviços especializados em PIC (onde só há exercício de PIC, sem cuidados biomédicos).

Tais modos foram encontrados nas cidades investigadas, em geral associados, com uns mais presentes que outros, conforme o contexto local, a história de iniciativas em PIC e as opções dos gestores municipais. Como no país, nessas cidades foi comum o protagonismo de profissionais praticantes de PIC que persistem e conseguem alguma institucionalização ${ }^{26}$. Mais recentemente, há algumas medidas de criação de política estaduais de PIC e municipais, estas últimas oficializando as opções locais de inserção. 
ISSN 2179-6750

Nota-se que dois dos modos acima (primeiro e segundo) estabelecem as PIC através de profissionais lotados nos serviços de APS. Ambos são limitados no sentido de que são atualmente muito raros os profissionais da APS ou da SF com competência em PIC, e raros os municípios que podem ou optam por incluir profissionais adicionais nas equipes de APS para exercer somente alguma PIC (e quando o fazem, isso ocorre em bem poucas equipes) ${ }^{26}$. A discussão sobre qual desses dois modos é melhor (para uma expansão progressiva, sustentável, substancial e a ser induzida das PIC, pressuposto de fundo deste texto), exige uma aproximação da realidade e das dificuldades da construção da APS no país, em que as PIC precisam ser cotejadas com as demais práticas de cuidado profissional. Como argumentamos antes ${ }^{26}$, a inserção de profissionais exclusivos de PIC de forma paralela ao cuidado generalista biomédico parece pouco sustentável de ser defendida como estratégia, devido a vários estrangulamentos graves da APS, dentre os quais: a) subfinanciamento federal e estadual, b) subdimensionamento da APS como um todo (muito poucas equipes de SF para o conjunto da população ou ao menos para $80 \%$ dela); b) proporcionalmente poucos profissionais capacitados para a atuação na APS (poucos médicos de família e comunidade com residência e enfermeiros com especialização em SF ou atenção básica); c) excesso de número de usuários vinculados as equipes de SF ou serviços de APS existentes, o que é agravado pela imensa iniquidade e desigualdade social brasileira, que produz mais frequentes e graves adoecimentos; d) (consequentemente) pouca qualificação clínica (biomédica) dos profissionais; e) dificuldade de concretização dos atributos da APS, sobretudo do acesso e da coordenação do cuidado (devido à pouca estruturação e articulação dos serviços especializados com a APS, além de seu subdimensionamento e pouca adequação ${ }^{27}$ ); f) consequente excesso de demanda por cuidado biomédico pressionando os profissionais.

Nessa realidade precária, como relacionar as PIC com a demanda por cuidado clínico em geral (suposto comumente como biomédico)? Caberia defender a criação de concursos ou introdução de profissionais nos centros de saúde que atuarão em paralelo ao cuidado clínico generalista biomédico no atual contexto da APS? Embora de um ponto de vista teórico seja defensável que haja outra RM ou alguma PIC acessíveis à população, parece-nos pouco sustentável essa defesa, que implica em contratação de mais profissionais para as mesmas equipes, tantos quantas forem as PIC que estejam regulamentadas como especialização, praticadas, portanto, de forma relativamente restrita e em geral por profissionais exclusivos delas (como homeopatia e acupuntura, mas poder-se-ia pensar em massagistas, quiropatas, osteopatas etc.). Além disso, esses profissionais são raros e a maior parte deles atuantes no setor privado, com formação para ele voltada. 
ISSN 2179-6750

Por outro lado, pode ser razoável defender e pensar que o SUS deve também sustentar a oferta de outras práticas não biomédicas de cuidado à saúde para a população, que fossem universalmente acessíveis e desvinculadas do cuidado biomédico, permitindo a escolha do tipo de abordagem pelos usuários. Isso significaria a oferta de outras PIC e RM na APS, que poderiam, talvez, (e nessa lógica deveriam) ser mais valorizadas e assim inseridas em paralelo ao cuidado convencional na APS. Se mesmo o cuidado biomédico não é acessível universalmente via APS, não haveria porque esperar pela sua ampliação e estruturação (como ocorreu em países de alta renda com sistemas de saúde públicos universais baseados na APS), para que se pluralizasse os recursos e as formas de cuidado. Apesar de haver algum acúmulo científico sobre as condições sensíveis à APS e a ela serem atribuídos vários impactos sobre a morbimortalidade, sendo implícito nesses resultados que a RM orientadora é a biomédica, isso não quer dizer que outros cuidados não pudessem também ter impacto, uma vez que isso é desconhecido e provavelmente não experimentado social e institucionalmente. Outrossim, a própria discussão sobre a determinação social do processo-saúde, que não é nova ${ }^{28}$, relativiza a potência e o impacto supostos do cuidado biomédico na morbimortalidade coletiva, de modo que sua presença disseminada e acessível como propõe o SUS e a literatura sobre a APS talvez não tivesse que ser tomada como condição sine qua non para a estruturação e construção da APS. Nessa lógica, a APS poderia ser entendida como um tanto desconectada da biomedicina, e compreendida em um sentido antropológico e social, como a estruturação de serviços comunitários de cuidado profissional à saúde organizados para prover curadores acessíveis aos cidadãos, como direito de cidadania, em que a biomedicina seria mais uma RM provedora de saberes, técnicas e recursos de cuidado, não necessariamente a única ordenadora da APS e do sistema de saúde, como é a tradição da saúde pública e dos sistemas nacionais de saúde públicos universais.

Neste caso, faria sentido que os praticantes fossem exclusivos de PIC, ao menos no caso de algumas delas, como as RM vitalistas e outras, de modo a que o usuário pudesse escolher a RM e ser tratado por um profissional com formação a melhor possível. Como as RM vitalistas mais conhecidas são mais centradas nas pessoas e integrais na sua abordagem ${ }^{29}$, e menos fragmentadas em subespecialidades, ao menos no Brasil, isso poderia significar um profissional ou curador de uma PIC ou RM a estar presente na APS em paralelo a equipe do cuidado biomédico.

Embora esse raciocínio faça talvez sentido para alguns praticantes ou especialistas em PIC, é mister reconhecer que está muito distante da realidade institucional e acadêmica, e mesmo do grosso dos profissionais da APS, formados e norteados pela RM biomédica. No contexto 
ISSN 2179-6750

acadêmico e institucional, essa discussão é vista mais como ampliação dos cuidados para terapias complementares, entendidas como complementares à biomedicina. Uma razão para ser considerado bem fundamentado esse centramento da APS na biomedicina é o reconhecimento de que na prática social, institucional e mesmo nos itinerários terapêuticos das pessoas, os profissionais biomédicos são reconhecidos, procurados e legitimados para estabelecer interpretações (diagnósticos) muito respeitadas nos casos dos adoecimentos mais graves, e seus recursos são muito valorizados em situações de urgência e emergência ou casos de grande gravidade clínica.

Essa situação de maior responsabilidade e legitimidade (e maior poder de manuseio de técnicas diagnósticas e terapêuticas mais valorizadas em situações extremas ou quase extremas, ou mais graves) dos profissionais e cuidados biomédicos pode ser considerada como um indicativo de que deve haver uma prioridade para a estruturação de uma rede de serviços de APS que coloque os recursos dessa RM a serviço das pessoas que dela precisam na população, como um direito de cidadania, uma vez que é ela que é principalmente procurada quando o caso é grave, urgente ou emergencial (ainda que as PIC também sejam complementarmente muito procuradas nesses casos, frente aos limites de efetividade e aos grandes efeitos adversos dos tratamentos biomédicos empregados). Nesse sentido, pode ser defendida uma prioridade para a estruturação de uma biomedicina generalista acessível universalmente na APS, levando em conta a experiência internacional a respeito. Assim, a ampliação de profissionais da APS teria como prioridade a RM biomédica na sua vertente generalista (MFC), sendo difícil sustentar a proliferação de praticantes exclusivos de PIC em paralelos as equipes já existentes de APS, como estratégia de expansão sustentável a curto prazo.

Outrossim, a inserção das PIC na APS através de seu exercício pelos profissionais realizadores do cuidado biomédico, ainda que com limites de competência e/ou contexto, teria como vantagem adicional o fato de que as mesmas podem ajudar substancialmente esses profissionais a reconhecerem e manejarem melhor várias mazelas de sua formação e tradição biomédica, e assim de suas práticas clínicas, melhorando seu espírito crítico para com os limites da nosologia e terapêutica biomédicas e para com a alta tolerância aos danos iatrogênicos de suas práticas. Além disso, melhoram a habilidade clínica para reconhecer e evitar situações de comum medicalização desnecessária e uso duvidoso de fármacos e exames complementares, ajudando no exercício do que se tem chamado na APS de prevenção quaternária: evitar danos potenciais e medicalização excessiva decorrentes do intervencionismo biomédico, protegendo os usuários de danos iatrogênicos e oferecendo alternativas eticamente aceitáveis a esses pacientes ${ }^{30}$. Pesquisa investigando a repercussão de um curso introdutório teórico prático de acupuntura (72 horas no 
ISSN 2179-6750

total) nas práticas de médicos da APS mostrou que essa vantagem adicional é significativa e se reflete na ampliação do olhar sobre processo saúde-doença e na ampliação dos recursos terapêuticos, bem como na criação de uma postura reflexiva sobre o uso de saberes biomédicos e complementares nos cuidados ${ }^{31}$.

No conjunto desses argumentos, parece-nos ser mais sustentável e defensável a estratégia de inserção progressiva das PIC na APS como habilidade e competência dos mesmos profissionais que já realizam o cuidado biomédico, os quais, geralmente, não tem competência em PIC, mas tem se mostrado no país e no mundo cada vez mais abertos e interessados nelas ${ }^{32}$. Tais profissionais teriam que ser iniciados em algumas PIC, o suficiente para fazerem uso seguro das mesmas, o que é viável em vários casos (a exemplo da auriculoterapia e acupuntura, acima citadas). Se possível, no caso das RM vitalistas, que pudessem ser nelas iniciados e estimulados a aprofundamento especializado, conforme o interesse e os resultados clínicos.

Essa estratégia não é rápida e demanda esforço institucional em várias frentes, incluindo pelo menos o estímulo sistemático à produção de ações de educação em serviço, na lógica da educação permanente ${ }^{33}$, e a introdução de competências em PIC na formação graduada e pósgraduada (residências em MFC e SF) dos profissionais da APS. Experiências, ainda que raras até agora, têm mostrado a potência do primeiro tipo de esforço ${ }^{26}$, em que ocorrem ações de educação em serviço contextualizadas e voltadas para os problemas práticos do trabalho na APS, voltadas a profissionais que aprendem e iniciam o exercício de PIC, com ampla aceitação de usuários ${ }^{34} \mathrm{e}$ progressiva legitimação institucional. O exemplo de Florianópolis é digno de nota a esse respeito. Há mais de cinco anos um processo de sensibilização, educação e estímulo progressivo ao uso de algumas PIC vem sendo desenvolvido, atravessou gestões municipais diversas, e prossegue baseando-se em parcerias e exploração da expertise de profissionais da própria instituição (principalmente envolvendo plantas medicinais, auriculoterapia, acupuntura, mas também práticas corporais e outras) ${ }^{35}$.

Vale salientar, que, para além de um recurso complementar ou alternativo de cuidado (modos de uso mais comuns no contexto institucional biomédico), as PIC na APS aparecem também como uma opção de cuidado anterior ao cuidado biomédico, como uma alternativa inicial adotada por parte dos profissionais e usuários, antecedendo o uso de recursos biomédicos típicos (fármacos), os quais ficam reservados para um fracasso ou agravamento da situação ${ }^{32}$. Esse uso é muito coerente com as características das PIC que as fazem adequadas à APS e muito usadas na autoatenção, e poderia ser muito mais explorado. Todavia, ele só pode ser explorado e desenvolvido, maximizando as virtudes das PIC, no modo de inserção primeiro, em que o 
ISSN 2179-6750

profissional biomédico aprende e exerce alguma(s) PIC, de modo a que a dupla profissional-usuário discuta e decida o tipo de cuidado mais adequado. A integração íntima das PIC na APS permeando o interior do processo de interpretação/decisão clínica exercida pelos próprios profissionais generalistas (biomédicos) apresenta essa especial vantagem, só existente nesse modo de inserção.

Por outro lado, essa inserção das PIC na APS restringe seu exercício pleno e especializado (no caso de PIC mais estruturadas e RM vitalistas), pois dificilmente as formações em serviço conseguem a extensão e profundidade comparáveis à formação especializada, mais longa e profunda, com carga teórico-prática muito maior. E esta formação quando existe ou existir nos serviços, em geral abrange(rá) uma pequena parcela dos profissionais, inicialmente pelo menos. Doutra parte, há que desenvolver nos serviços do SUS e instituições formadoras polos de excelência, como qualquer outra área, que produzam e reproduzam saber e pesquisa, que ensinem e disseminem competências a serem absorvidas pelos generalistas da APS e a serem exercidas em serviços especializados, como dissemos, para contribuir com o cuidado dos casos mais graves. Por isso, defendemos a associação do primeiro modo de inserção descrito acima com o terceiro, em que há matriciamento em PIC por profissionais especializados (ou equivalentes), devido a potencialidade de sinergia entre eles ${ }^{26}$.

Os especialistas em PIC (como, aliás, os demais especialistas em geral), devem praticar seus cuidados atendendo usuários, mas também devem matriciar equipes de APS para serem por elas coordenados e em conjunto fazer educação permanente. No caso das PIC, essa educação em serviço pode ser muito explorada, já que quase inexiste competência generalista baseada em PIC na APS de forma disseminada, o que é um projeto a ser construído, que tem sinais e bases teóricas e empíricas consistentes para ser não só possível como desejável e factível, conforme argumentamos acima.

Todavia, o matriciamento em PIC precisaria assumir (como todos os outros) o caráter duplo e pleno do apoio matricial: o exercício de cuidado em PIC aos usuários, para explorar e exercer as PIC nos usuários referenciados, e também o apoio às equipes, com especial atenção para educação permanente e iniciação em PIC, dado que muitas delas, ao menos em parte, e progressivamente, podem ser aprendidas e exercidas pelos generalistas da APS. Assim, todos os servidos especializados (em PIC e nas especialidades biomédicas e outras profissões da saúde) deveriam atuar com o matriciamento como parte de sua rotina, acabando com o isolamento nefasto dos serviços especializados em relação a APS. Argumentação e fundamentação para essa ideia ainda pouco discutida e compreendida, que não cabem neste momento, estão em Tesser ${ }^{36}$ e Tesser e Poli Neto ${ }^{27}$. Essa é a razão pela qual defendemos a conversão progressiva dos serviços especializados em geral (e também em PIC) em equipes e serviços matriciadores, quebrando o isolamento entre 
ISSN 2179-6750

especialistas e generalistas.

As PIC e as RM vitalistas e seus especialistas de serviços especializados (ou equipes de apoio) teriam, na perspectiva acima esboçada, uma relação com os profissionais da APS de natureza similar à relação exercida pelas especialidades médicas e não-médicas não generalistas (no sentido específico de não atenderem a qualquer pessoa de qualquer idade com qualquer tipo de problema ao longo de grande tempo com acesso fácil e abordagem familiar e comunitária): os generalistas da APS devem exercer e praticar saberes/técnicas de PIC (assim como de outras especialidades) na medida em que haja relevância, segurança, prevalência significativas, adequação tecnológica e social e efetividade no contexto local. Isso tudo (relevância, segurança, adequação, aceitação social e efetividade) parece estar garantido para várias PIC, ao menos na versão de profissionais já competentes e usuários (e em parte com razoáveis evidências corroborando essa percepção empírica), e seria o caso de se construir e sistematizar um arcabouço generalista de saberes e técnicas de PIC, análogo ao que a MFC fez com o conjunto das especialidades médicas: escrutinar e adaptar o que de relevante, útil, adequado e eficaz das várias PIC pode e deve ser colocado a disposição da população, a ser ensinado e praticado pelos profissionais da APS. Um desafio cheio de obstáculos (dada a incipiente institucionalização de várias PIC, sua pequenez e dispersão social, a não regulamentação e pouco reconhecimento formais para vária delas), mas que não deve por sua dificuldade obscurecer sua relevância e potencialidade. Essa analogia ajuda a iluminar também os modos de inserção das PIC, se considerarmos inaceitáveis para a construção da rede de serviços do SUS esquemas de isolamento dos serviços especializados similares a tantos que já existem, exigindo das equipes e serviços especializados matriciamento e íntima articulação e trabalho colaborativo com a APS.

\section{Conclusão}

A inserção e expansão das PIC no SUS têm ocorrido sem direção definida, capitaneada pelas iniciativas municipais, que as inserem majoritariamente na APS. A discussão acima pode contribuir para a construção de estratégias nacionais e regionais ou mesmo municipais, em cidades maiores, para progressiva inserção de várias PIC no SUS, de forma integrada com a APS, através de seus próprios profissionais, matriciados por serviços e equipes de profissionais especialistas em PIC que aceitem o desafio de socializar suas técnicas e saberes, e que contribuam com sua expertise em ações de educação permanente e cuidado colaborativo e compartilhado, bem como atendam os usuários com PIC. Provavelmente, se as PIC participarem de um processo em que sinergicamente os vários serviços do SUS se articulem progressivamente em rede coordenada pela APS e 
ISSN 2179-6750

contribuam para a construção da melhoria do cuidado generalista na SF, através de educação permanente e matriciamento, as PIC poderão crescer em significação e presença no SUS, tanto praticadas prioritariamente pelos generalistas da APS (SF) como por profissionais de equipes de apoio a SF e serviços especializados (que devem realizar matriciamento também).

\section{Referências}

1. World Health Organization. WHO traditional medicine strategy: 2014-2023. Geneva: WHO; 2013.

2. Luz MT, Barros NF, organizadores. Racionalidades médicas e práticas integrativas em saúde: estudos teóricos e empíricos. Rio de Janeiro: UERJ/IMS/LAPPIS; 2012.

3. Kuhn TS. A estrutura das revoluções científicas. São Paulo: Perspectiva; 1987.

4. Kuhn TS. A tensão essencial. Lisboa: Edições 70; 1989.

5. Fleck L. Gênese e desenvolvimento de um fato científico. Belo Horizonte: Fabrefactum; 2010.

6. Tesser CD. Produção de saber, racionalidades médicas e cuidado: ideias iniciais. In: Nascimento MC, Nogueira MI, organizadores. Intercâmbio solidário de saberes em saúde: racionalidades médicas e práticas integrativas e complementares. 1a ed. Vol. 1. São Paulo: Hucitec; 2013. p. 80-105.

7. Starfield B. Atenção primária: equilíbrio entre necessidades de saúde, serviços e tecnologia. Brasília, DF: UNESCO, Ministério da Saúde; 2002. Disponível em: http://unesdoc.unesco.org/images/0013/001308/130805por.pdf

8. Menendez EL. Modelos de atención de los padecimientos: de exclusiones teóricas y articulaciones prácticas. Cienc Saude Colet. 2003;8(1):185-207. http://dx.doi.org/10.1590/S1413-81232003000100014.

9. Lopes NM. Automedicação, saberes e racionalidades leigas em mudança. Rev Crit Cienc Sociais. 2007 out;8(1):185-207. http://dx.doi.org/10.1590/S1413-81232003000100014.

10. Silva LF, Alves F. Compreender as racionalidades leigas sobre saúde e doença. Physis. 2011;21(4):1207-29. http://dx.doi.org/10.1590/S0103-73312011000400003.

11. Gori L, Firenzuoli, F. Ear acupuncture in European traditional medicine. Evid Based Complement Alternat Med. 2007 Sep;4(Suppl 1):13-6. http://dx.doi.org/10.1093/ecam/nem106.

12. Abbate S. Chinese auricular acupuncture. Boca Raton: CRC Press; 2016.

13. Nogier P. Traité d'auriculothérapie. Moulin-lès-Metz: Maisonneuve;1969. 
ISSN 2179-6750

14. Oleson TD. Bases neurofisiológicas da acupuntura auricular. In: Stux G, Hammerschalg R, editores. Acupuntura clínica: bases científicas. São Paulo: Manole; 2005. p. 107-24.

15. Levin JS, Jonas WB, editores. Tratado de medicina complementar e alternativa. São Paulo: Manole; 2001.

16. Giacoia Junior O. Hans Jonas: O princípio responsabilidade: ensaio de uma ética para a civilização tecnológica. Cad Hist Filos Cienc. 1996;6(2):63-84.

17. Clarke AE, Mamo L, Fosket JR, Fishman JR, Shim JK. Biomedicalization: Technoscience, Health, and Illness in the U.S. Durham, NC: Duke University Press; 2010.

18. Astin JA. Why patients use alternative medicine: results of a national study. JAMA. 1998 May 20;279(19):1548-53. http://dx.doi.org/10.1001/jama.279.19.1548.

19. Andrade JT. Medicinas alternativas e complementares: experiência, corporeidade e transformação. Salvador: EDUFBA; 2006.

20. Tesser CD, Barros NF. Medicalização social e medicina alternativa e complementar: pluralização terapêutica do Sistema Único de Saúde. Rev Saude Publica. 2008;42(5):91420. http://dx.doi.org/10.1590/S0034-89102008000500018.

21. Stewart MA, McWhinney IR, Buck CW. The doctor/patient relationship and its effect upon outcome. J R Coll Gen Pract. 1979 Feb;29(199):77-82.

22. Beckman HB, Frankel RM. The effect of physician behaviour on the collection of data. Ann Intern Med. 1984 Nov;101(5):692-6.

23. Schulberg HC, Burns BJ. Mental disorders in primary care. Gen Hosp Psychiatry. 1988 Mar;10(2):79-87.

24. Starfield B, Wray C, Hess K, Gross R, Birk PS, D'Lugoff BC. The influence of patientpractitioner agreement on outcome of care. Am J Public Health. 1981 Feb;71(2):127-31.

25. Siegel P, Barros NF. O que é a oncologia integrativa?. Cad Saude Colet. 2013;21(3):348-54. http://dx.doi.org/10.1590/S1414-462X2013000300018.

26. Sousa IMC, Tesser CD. Medicina Tradicional e Complementar no Brasil: inserção no Sistema Único de Saúde e integração com a atenção primária. Cad Saude Publica. 2017;33(1):e00150215. http://dx.doi.org/10.1590/0102-311x00150215.

27. Tesser CD, Poli Neto P. Atenção especializada ambulatorial no Sistema Único de Saúde: para superar um vazio. Cienc Saude Colet. 2017;22(3):941-51. http://dx.doi.org/10.1590/1413-81232017223.18842016.

28. Mckeown T. El papel de la medicina: sueño, espejismo o némesis?. México, DF: Siglo Veintiuno Editores; 1982. 
ISSN 2179-6750

29. Tesser CD, Luz MT. Racionalidades médicas e integralidade. Cienc Saude Colet. 2008;13(1):195-206. http://dx.doi.org/10.1590/S1413-81232008000100024.

30. Norman AH, Tesser CD. Prevenção quaternária na atenção primária à saúde: uma necessidade do Sistema Único de Saúde. Cad Saude Publica. 2009;25(9)2012-20. http://dx.doi.org/10.1590/S0102-311X2009000900015.

31. Moré AOO. Educação permanente em acupuntura: análise de um processo educativo e suas repercussões na prática de médicos da Atenção Primária à Saúde de Florianópolis [tese]. Florianópolis: Universidade Federal de Santa Catarina; 2016. https://repositorio.ufsc.br/xmlui/handle/123456789/172583

32. Thiago SCS, Tesser CD. Percepção de médicos e enfermeiros da Estratégia de Saúde da Família sobre terapias complementares. Rev Saude Publica. 2011;45(2):249-57.

33. Peduzzi M, Guerra DAD, Braga CP, Lucena FS, Silva JAM. Atividades educativas de trabalhadores na atenção primária: concepções de educação permanente e de educação continuada em saúde presentes no cotidiano de Unidades Básicas de Saúde em São Paulo. Interface (Botucatu). 2009 set;13(30):121-34.

34. Faqueti A. Medicinas alternativas e complementares na atenção primária à saúde: perspectiva de usuários em Florianópolis/SC [dissertação]. Florianópolis: Universidade Federal de Santa Catarina; 2014. https://repositorio.ufsc.br/xmlui/handle/123456789/129053

35. Santos MC, Tesser CD. Um método para a implantação e promoção de acesso às Práticas Integrativas e Complementares na Atenção Primária à Saúde. Cienc Saude Colet. 2012;17(11):3011-24. http://dx.doi.org/10.1590/S1413-81232012001100018.

36. Tesser CD. Núcleos de Apoio à Saúde da Família, seus potenciais e entraves: uma interpretação a partir da atenção primária à saúde. Interface (Botucatu). 2017 jul;21(62):565-78. http://dx.doi.org/10.1590/1807-57622015.0939. 